\title{
Recovery efficiencies for Burkholderia thailandensis from various aerosol sampling media
}

\author{
Paul Dabisch *, Kristin Bower, Brandi Dorsey and Loni Wronka \\ National Biodefense Analysis and Countermeasures Center, US Department of Homeland Security, Frederick, MD, USA
}

\section{Edited by:}

Margaret L. M. Pitt, United States Army Medical Research Institute of Infectious Diseases, USA

\section{Reviewed by:}

Martin I. Voskuil, University of

Colorado Denver, USA

Larry E. Bowen, Southern Research Institute, USA

\section{${ }^{*}$ Correspondence:}

Paul Dabisch, National Biodefense Analysis and Countermeasures

Center, US Department of

Homeland Security, Frederick, MD

21702, USA.

e-mail:paul.dabisch@nbacc.dhs.gov
Burkholderia thailandensis is used in the laboratory as a surrogate of the more virulent B. pseudomallei. Since inhalation is believed to be a natural route of infection for B. pseudomallei, many animal studies with $B$. pseudomallei and $B$. thailandensis utilize the inhalation route of exposure. The aim of the present study was to quantify the recovery efficiency of culturable $B$. thailandensis from several common aerosol sampling devices to ensure that collected microorganisms could be reliably recovered post-collection. The sampling devices tested included $25 \mathrm{~mm}$ gelatin filters, $22 \mathrm{~mm}$ stainless steel disks used in Mercer cascade impactors, and two types of glass impingers. The results demonstrate that while several processing methods tested resulted in significantly lower physical recovery efficiencies than other methods, it was possible to obtain culturable recovery efficiencies for $B$. thailandensis and physical recovery efficiencies for $1 \mu \mathrm{m}$ fluorescent spheres of at least 0.95 from all of the sampling media tested given an appropriate sample processing procedure. The results of the present study also demonstrated that the bubbling action of liquid media in all-glass impingers (AGIs) can result in physical loss of material from the collection medium, although additional studies are needed to verify the exact mechanisms involved. Overall, the results of this study demonstrate that the collection mechanism as well as the post-collection processing method can significantly affect the recovery from and retention of culturable microorganisms in sampling media, potentially affecting the calculated airborne concentration and any subsequent estimations of risk or dose derived from such data.

Keywords: Burkholderia thailandensis, aerosol sampling, culturable recovery efficiency, physical recovery efficiency, aerosol, inhalation

\section{INTRODUCTION}

Many different sampling devices are available for the collection of airborne microorganisms. The sampling process involves entry of airborne microorganisms into the sampler inlet, transport to a sampling medium, collection by the sampling medium, retention in the sampling medium in a culturable state for the duration of sampling, recovery from the sampling medium following termination of the sampling, and determination of the concentration of microorganisms present. The total efficiency of the sampling process can be expressed as the product of the individual efficiencies associated with each of the various components of the sampling process (Brockman, 2011; Reponen et al., 2011). Knowledge of the total efficiency of the sampling process, as well as the efficiencies of the various components, is important in studies where the absolute concentration of airborne material is needed, such as inhalation dosing studies. Inaccurate assumptions regarding of these efficiency values, or errors in their estimation, have the potential to affect the calculated airborne concentration of microorganisms and any subsequent estimations of risk or dose derived from such data.

The recovery efficiency quantifies the recovery of collected material from a given sampling medium after it has been collected. As noted previously, the recovery efficiency of collected material from a sampling medium contributes to the total efficiency of the sampling process. Two separate terms, the physical recovery efficiency and the culturable recovery efficiency are often used to describe losses due to physical processes and decreases in culturability, respectively. Decreases in the physical recovery efficiency are often estimated with the aid of fluorescent tracers, and can be attributed to the physical loss of material during post-collection processing. Decreases in the culturable recovery efficiency are often associated with a loss of culturability of the collected microorganisms due to stresses associated with the post-collection processing (Zhao et al., 2011).

B. pseudomallei, the causative agent of melioidosis, is a gram negative rod shaped bacterium that is found in soil and water in areas where it is endemic, including many of the countries of Southeast Asia, and recently Europe and South America (Inglis and Sagriapanti, 2006). Melioidosis in humans occurs in individuals who have been in contact with contaminated surface water and soil (Cheng and Currie, 2005). Inhalation of airborne bacteria is also believed to be a route of exposure in areas where monsoonal flooding and high winds occur (Currie, 2008). Therefore, several studies with aerosolized B. pseudomallei have been conducted to develop animal models of inhalational melioidosis for use in therapeutic efficacy testing (Sivalingam et al., 2008; 
Tan et al., 2008; Rozak et al., 2010). However, since B. pseudomalle $i$ is a CDC Category B biological threat agent, work with this organism is highly regulated and requires special laboratory facilities. Given these restrictions, Burkholderia thailandensis, which is genetically similar to $B$. pseudomallei, is commonly used in the laboratory as a surrogate of the more virulent $B$. pseudomalle $i$ (Haraga et al., 2008; West et al., 2008).

Previous studies utilizing inhalational murine models of either B. pseudomallei or B. thailandensis infection have calculated inhaled doses calculated either by assaying lung tissue post exposure (West et al., 2008) or based on the measured airborne bacterial concentration determined using impingers (Sivalingam et al., 2008; Tan et al., 2008; Morici et al., 2010; Rozak et al., 2010). However, none of the studies that utilized impingers discuss the efficiency of the sampling devices with aerosolized Burkholderia species. A recent study compared the total sampling efficiency and the retention efficiency of several aerosol sampling devices with B. pseudomallei (Dabisch et al., 2012), demonstrating the equivalence of the total sampling efficiencies for the Mercer cascade impactor, gelatin filters, and an all glass impinger across a range of concentrations for aerosolized B. pseudomallei. However, the retention efficiency was shown to differ between gelatin filters and an all glass impinger, demonstrating that while the total sampling efficiencies were similar between the devices, the efficiencies of the various components of the sampling process were not. Given this previous data, the aim of the present study was to estimate the efficiency for another component of the sampling process, namely the physical and culturable recovery efficiencies of $B$. thailandensis, from several commonly used sampling media, specifically $25 \mathrm{~mm}$ gelatin filters, greased $25 \mathrm{~mm}$ stainless steel disks from a Mercer impactor, and jars from both an all-glass impinger (AGI) and an SKC Biosampler, using a variety of different recovery processes. These data will useful to inform sampling paradigms in future inhalation studies, as well as for correction of concentration, and subsequent dose calculations in inhalation studies.

\section{MATERIALS AND METHODS \\ PREPARATION OF Burkholderia thailandensis}

Tryptic soy broth (TSB; PN 290613, BD Biosciences, Franklin Lakes, NJ, USA) was inoculated with frozen $B$. thailandensis stock culture (ATCC 700388) in a baffled flask, and incubated for approximately $18 \mathrm{~h}$ at $35^{\circ} \pm 2{ }^{\circ} \mathrm{C}$ with shaking. Dilutions were prepared in fresh TSB.

\section{OVERVIEW OF RECOVERY STUDIES}

Both the physical recovery efficiency and the culturable recovery efficiency were estimated for several different sampling media using a variety of different recovery processes, details of which are provided in the following sections. The collection media tested included $25 \mathrm{~mm}$ gelatin filters (PN\#225-9551, SKC, Inc., Eighty-Four, PA, USA), greased $22 \mathrm{~mm}$ stainless steel disks from a Mercer impactor (Intox Products, Moriarty, NM, USA), and jars from both a Model 7541 AGI (Ace Glass Inc., Vineland, NJ, USA), and an SKC Biosampler (PN\#225-9596, SKC, Inc., EightyFour, PA, USA). The physical recovery efficiency was estimated by comparing the recovery of $1 \mu \mathrm{m}$ fluorescent melamine beads
(Thermo Scientific, Fremont, CA, USA) from processed samples to recovery from control samples. Unlike a biological organism, a non-biological tracer, such as fluorescent beads, is not subject to losses in viability associated with aerosolization and sampling. One $\mu \mathrm{m}$ fluorescent melamine beads were chosen since they are approximately the same size and shape of the bacterium used for estimating culturable recovery efficiency, B. thailandensis. The culturable recovery efficiency was estimated by comparing the recovery of $B$. thailandensis from processed samples to the recovery from control samples. Estimation of the culturable recovery efficiency was not performed using processing methods that had physical recovery efficiencies that were significantly less than the other processes.

\section{RECOVERY FROM $25 \mathrm{~mm}$ GELATIN FILTERS}

To estimate recovery of $1 \mu \mathrm{m}$ fluorescent melamine beads from $25 \mathrm{~mm}$ gelatin filters, $10 \mu \mathrm{l}$ of water containing $1 \mu \mathrm{m}$ fluorescent melamine beads was spotted onto the surface of a $25 \mathrm{~mm}$ gelatin filter located in the bottom of a $50 \mathrm{ml}$ conical tube. The filter was allowed to sit for $10 \mathrm{~min}$ and was then processed. Four different processing methodologies (10 replicates each) were evaluated: (1) the addition of $10 \mathrm{ml}$ of water at room temperature, and vortexing for $3 \mathrm{~min}$ to dissolve the filter; (2) the addition of $10 \mathrm{ml}$ of water at $35^{\circ} \mathrm{C}$, and vortexing for $3 \mathrm{~min}$ to dissolve the filter; (3) the addition of $10 \mathrm{ml}$ of water at $35^{\circ} \mathrm{C}$, and vortexing for $30 \mathrm{~s}$ to dissolve the filter; and (4) the addition of $10 \mathrm{ml}$ of water at $35^{\circ} \mathrm{C}$, and vortexing for $10 \mathrm{~s}$ to dissolve the filter. Sample fluorescence was quantified using a SpectraMax M5 Fluorescence Reader (Molecular Devices, Sunnyvale, CA, USA) with an excitation wavelength of $460 \mathrm{~nm}$ and an emission wavelength of $520 \mathrm{~nm}$.

The methodology to estimate the recovery of $B$. thailandensis from $25 \mathrm{~mm}$ gelatin filters was identical to that used for the recovery of $1 \mu \mathrm{m}$ fluorescent melamine beads, except that TSB was used in place of water, and the bacterial content of samples was determined by triplicate plating samples on tryptic soy agar (TSA; PN 221283, BD Biosciences, Franklin Lakes, NJ, USA) plates with enumeration following a $48 \mathrm{~h}$ incubation period. Recovery processes that resulted in a physical recovery efficiency that was significantly less than that of the others were not tested with B. thailandensis.

Control tubes contained a clean gelatin filter to which either $10 \mathrm{ml}$ of either TSB (for bacterial samples) or water (for bead samples) at the appropriate temperature was added. Each tube was processed by one of the methodologies described above to dissolve the filter. Following dissolution of the filter, $10 \mu \mathrm{l}$ containing either bacteria or beads was added to the liquid in the tube, the tube was vortexed for $3 \mathrm{~s}$ to mix the contents, and the contents were assayed as described previously. For $1 \mu \mathrm{m}$ fluorescent melamine beads, the physical recovery efficiency from the spiked filters was expressed as the ratio of the amount of fluorescence recovered from the spiked filters to the average amount of fluorescence recovered from the triplicate control tubes. For $B$. thailandensis, the culturable recovery efficiency from the spiked filters was expressed as the ratio of the number of colonies recovered from the spiked filters to the average number of colonies recovered from the triplicate control tubes. 


\section{RECOVERY FROM 22 mm STAINLESS STEEL DISKS}

Prior to recovery testing, the stainless steel disks were greased by working a small amount of silicone grease (Intox Products, Moriarty, NM, USA) between the fingers of a gloved hand and then rolling a finger over the center if a disk, leaving a thin fingerprint of silicone grease, as is commonly done with cascade impactors to prevent bounce of particles following impaction on the disk. To estimate recovery of $1 \mu \mathrm{m}$ fluorescent melamine beads from greased $22 \mathrm{~mm}$ stainless steel disks, $10 \mu \mathrm{l}$ of water containing $1 \mu \mathrm{m}$ fluorescent melamine beads was placed on the center of a greased $22 \mathrm{~mm}$ stainless steel disk. The disk was allowed to sit for $10 \mathrm{~min}$, and was then placed into a $50 \mathrm{ml}$ conical tube containing $4 \mathrm{~mL}$ of water at room temperature. Three different recovery processes (10 replicates each) were evaluated: (1) vortexing for $3 \mathrm{~min}$ to re-suspend the beads; (2) vortexing for $30 \mathrm{~s}$ to re-suspend the beads; and (3) vortexing for $10 \mathrm{~s}$ to re-suspend the beads. Sample fluorescence was quantified using a SpectraMax M5 Fluorescence Reader with an excitation wavelength of $460 \mathrm{~nm}$ and an emission wavelength of $520 \mathrm{~nm}$.

The methodology to estimate the recovery of $B$. thailandensis from $22 \mathrm{~mm}$ stainless steel disks was identical to that used for the recovery of $1 \mu \mathrm{m}$ fluorescent melamine beads, except that TSB was used in place of water, and the bacterial content of samples was determined by triplicate plating onto TSA plates with enumeration following a $48 \mathrm{~h}$ incubation period. Recovery processes that resulted in a physical recovery efficiency that was significantly less than the others were not tested with $B$. thailandensis.

Control tubes contained a clean greased stainless steel disk to which $4 \mathrm{ml}$ of either TSB (for bacterial samples) or water (for bead samples) at room temperature was added. Each tube was vortexed for one of the durations described above, after which $10 \mu \mathrm{l}$ containing either bacteria or beads was added to the liquid in the tube. The tube was then vortexed for $3 \mathrm{~s}$ to mix the contents, and the contents were assayed as described previously. For $1 \mu \mathrm{m}$ fluorescent melamine beads, the physical recovery efficiency from the spiked stainless steel disks was expressed as the ratio of the amount of fluorescence recovered from the spiked disks to the average amount of fluorescence recovered from the triplicate control tubes. For $B$. thailandensis, the culturable recovery efficiency from the spiked disks was expressed as the ratio of the number of colonies recovered from the spiked disks to the average number of colonies recovered from the triplicate control tubes.

\section{RECOVERY FROM IMPINGER JARS}

To estimate recovery of $1 \mu \mathrm{m}$ fluorescent melamine beads from impinger jars, a stock suspension of $1 \mu \mathrm{m}$ fluorescent melamine beads in water was prepared. The baseline fluorescence was determined using a SpectraMax M5 Fluorescence Reader. Ten mL of the stock suspension was pipetted into the jar of either an AGI or Biosampler. The jar was allowed to sit for $10 \mathrm{~min}$ after which the suspension was decanted into a clean $15 \mathrm{ml}$ conical tube, and the fluorescence of the sample was measured using a SpectraMax M5 Fluorescence Reader. Ten replicates were performed with each type of jar. The physical recovery efficiency of each jar type was calculated as the ratio of the amount of material recovered from the jar (product of concentration of material in jar and volume recovered from jar) to the amount of material initially pipetted into each jar (product of the volume initially pipetted into the jar and the stock concentration).

The methodology to estimate the recovery of $B$. thailandensis from impinger jars was identical to that used for the recovery of $1 \mu \mathrm{m}$ fluorescent melamine beads, except that TSB was used in place of water, and the bacterial content of samples was determined by triplicate plating onto TSA plates with enumeration following a $48 \mathrm{~h}$ incubation period. The culturable recovery efficiency from a jar was expressed as the ratio of the number of colonies recovered from the jar (product of bacterial concentration in jar and volume recovered from jar) to the number of colonies initially pipetted into the jar (product of the volume initially pipetted into the jar and the stock bacterial concentration).

Since both the AGI and the Biosampler swirl/bubble the liquid media during the collection process, the ability of the sampler to retain material already present in the liquid media during the collection process was also estimated. A stock suspension of $1 \mu \mathrm{m}$ fluorescent melamine beads in water was prepared, and the baseline fluorescence was determined using a SpectraMax M5 Fluorescence Reader. For AGI jars, either 10 or $20 \mathrm{ml}$ of the stock suspension was pipetted into the jar. For SKC jars, either 15 or $20 \mathrm{ml}$ of the stock suspension was pipetted into the jar. Each jar was then attached to the other components of the sampler, and the sampler was connected to a vacuum source to initiate air flow through the sampler. The duration of air flow through the sampler was $10 \mathrm{~min}$. The inlet of the sampler remained open to room air. Following termination of air flow through the sampler, the remaining suspension was decanted into a clean conical tube, and the fluorescence of the sample was measured using a SpectraMax M5 Fluorescence Reader. The physical retention efficiency for each jar was calculated as the ratio of the amount of material recovered from the jar (product of concentration of material in jar and volume recovered from jar) to the amount of material initially pipetted into each jar (product of the volume initially pipetted into the jar and the stock concentration).

The retention of $B$. thailandensis was only estimated in AGI jars. The methodology was identical to that used for estimating the retention of $1 \mu \mathrm{m}$ fluorescent melamine beads, except that only $10 \mathrm{ml}$ of TSB containing bacteria was used, and the bacterial content of samples was determined by triplicate plating onto TSA plates with enumeration following a $48 \mathrm{~h}$ incubation period. The duration of air flow through the sampler was $10 \mathrm{~min}$. The culturable retention efficiency for the AGI jar containing $10 \mathrm{ml}$ of media was calculated as the ratio of the number of colonies recovered from the jar (product of bacterial concentration in jar and volume recovered from jar) to the number of colonies initially pipetted into each jar (product of the volume initially pipetted into the jar and the stock bacterial concentration).

\section{STATISTICS}

All statistical analyses were done using GraphPad Prism (Version 5.04, 2010). Comparisons of different sampling devices and recovery processes were done using One-Way analysis of variance with a Bonferonni post-test. All values are presented as 
mean \pm standard deviation. An alpha value of 0.05 was used as the criterion for statistical significance.

\section{RESULTS}

\section{PHYSICAL RECOVERY OF $1 \mu \mathrm{m}$ FLUORESCENT MELAMINE BEADS}

The physical recovery efficiency of $1 \mu \mathrm{m}$ fluorescent melamine beads from $25 \mathrm{~mm}$ gelatin filters was assessed using four different processing methods, and the results are shown in Figure 1. The physical recovery efficiency of fluorescent beads from gelatin filters following $3 \mathrm{~min}$ of vortexing in room temperature water $(0.52 \pm 0.14, n=10)$ was significantly less $(P<0.0001)$ than the other three processing methods evaluated $\left(10 \mathrm{~s}, 35^{\circ} \mathrm{C}\right.$ : $0.97 \pm 0.03, n=10 ; 30 \mathrm{~s}, 35^{\circ} \mathrm{C}: 0.95 \pm 0.04, n=10 ; 3 \mathrm{~min}, 35^{\circ} \mathrm{C}$ : $0.96 \pm 0.02, n=10)$.

The physical recovery efficiency of $1 \mu \mathrm{m}$ fluorescent melamine beads from $22 \mathrm{~mm}$ stainless steel disks was assessed using three different processing methods, and the results are shown in Figure 2. The physical recovery efficiency of fluorescent beads from stainless steel disks was inversely proportional to the duration of vortexing: $10 \mathrm{~s}=1.05 \pm 0.06(n=10) ; 30 \mathrm{~s}=0.85 \pm 0.11$ $(n=10) ; 3 \mathrm{~min}=0.23 \pm 0.08(n=10)$. The physical recovery efficiencies for all three recovery methods were significantly different from each other $(P<0.0001)$. Additionally, it was also noted that the walls of the conical tube became more opaque as the duration of vortexing increased.

The physical recovery efficiencies of $1 \mu \mathrm{m}$ fluorescent melamine beads from impinger jars were assessed and the results are shown in the top of Figure 3. The physical recovery efficiency of fluorescent beads from both jars types were

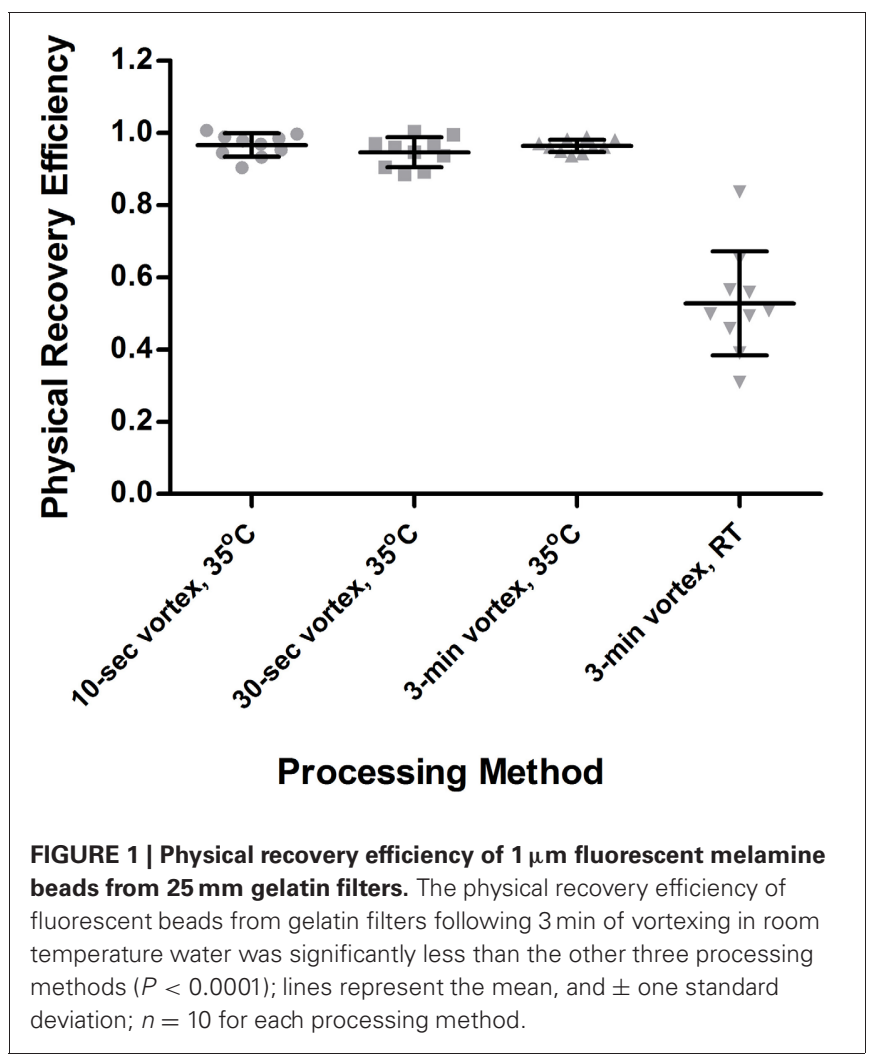

similar, with a physical recovery efficiency from the AGI jars of $1.03 \pm 0.03(n=10)$, and a physical recovery efficiency from the SKC Biosampler jars of $1.03 \pm 0.01(n=10)$.

The physical retention efficiencies of impinger jars with $1 \mu \mathrm{m}$ fluorescent melamine beads were assessed and the results are shown in the bottom of Figure 3. The physical retention efficiencies of the SKC jars filled with $15(n=9)$ and $20 \mathrm{ml}(n=8)$ of media containing $1 \mu \mathrm{m}$ fluorescent beads were $0.97 \pm 0.07$ and $1.07 \pm 0.09$, respectively. The physical retention efficiencies of the AGI jars filled with $10(n=8)$ and $20 \mathrm{ml}(n=3)$ of media containing $1 \mu \mathrm{m}$ fluorescent beads were $0.16 \pm 0.06$ and $0.57 \pm 0.19$, respectively. Additionally, following decanting of the liquid media, a residue was visible on the inside of the AGI jar above where the air-liquid interface had been.

\section{CULTURABLE RECOVERY OF $\boldsymbol{B}$. thailandensis}

The culturable recovery efficiency of $B$. thailandensis from gelatin filters using three different processing methods, stainless steel disks, and two types of impinger jars was assessed, and the results are shown in Figure 4. Culturable recovery efficiency from filters processed with room temperature TSB, and stainless steel disks vortexed for either $30 \mathrm{~s}$ or $3 \mathrm{~min}$, were not assessed since the physical recovery of $1 \mu \mathrm{m}$ fluorescent melamine beads using these recovery processes was significantly less than the other methods tested. The culturable recovery efficiency of $B$. thailandensis from any of the sampling media and recovery processes tested were not significantly different from one another $(P=0.1569 ; n=10$ for each type of media): AGI Jar $=0.95 \pm 0.07$; SKC Biosampler Jar $=0.95 \pm 0.12$; Stainless Steel Disk ( 10 s vortex $)=0.97 \pm 0.14$; Gelatin filter with $10 \mathrm{~s}$ vortex @ $35^{\circ} \mathrm{C}=0.98 \pm 0.15$; Gelatin filter with 30 s vortex @ $35^{\circ} \mathrm{C}=1.06 \pm 0.08$; Gelatin filter with $3 \mathrm{~min}$

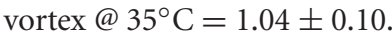

The culturable retention efficiency of AGI jars with $B$. thailandensis was also assessed. The culturable retention efficiency of the

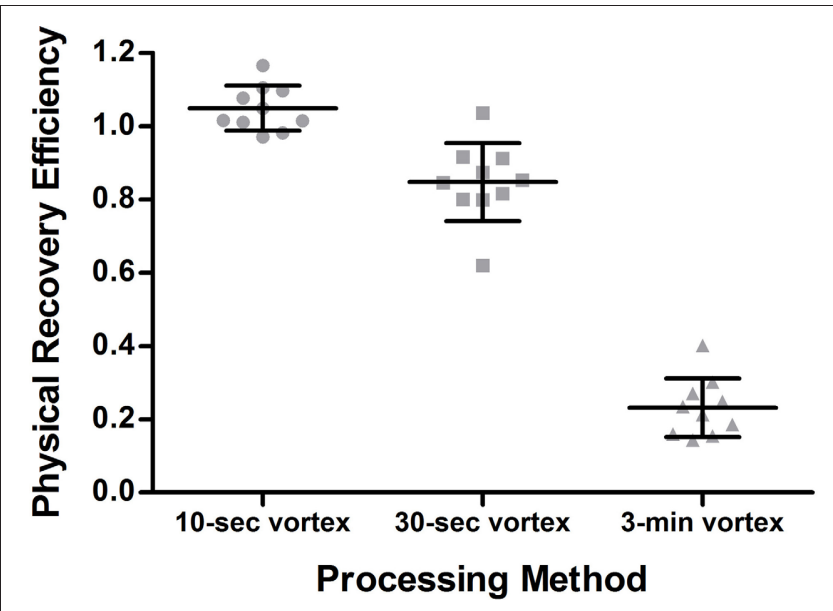

FIGURE 2 | Physical recovery efficiency of $1 \mu \mathrm{m}$ fluorescent melamine beads from $22 \mathrm{~mm}$ stainless steel disks. The physical recovery efficiency of fluorescent beads from stainless steel disks was significantly different for all three processing methods tested $(P<0.0001)$; lines represent the mean, and \pm one standard deviation; $n=10$ for each method. 


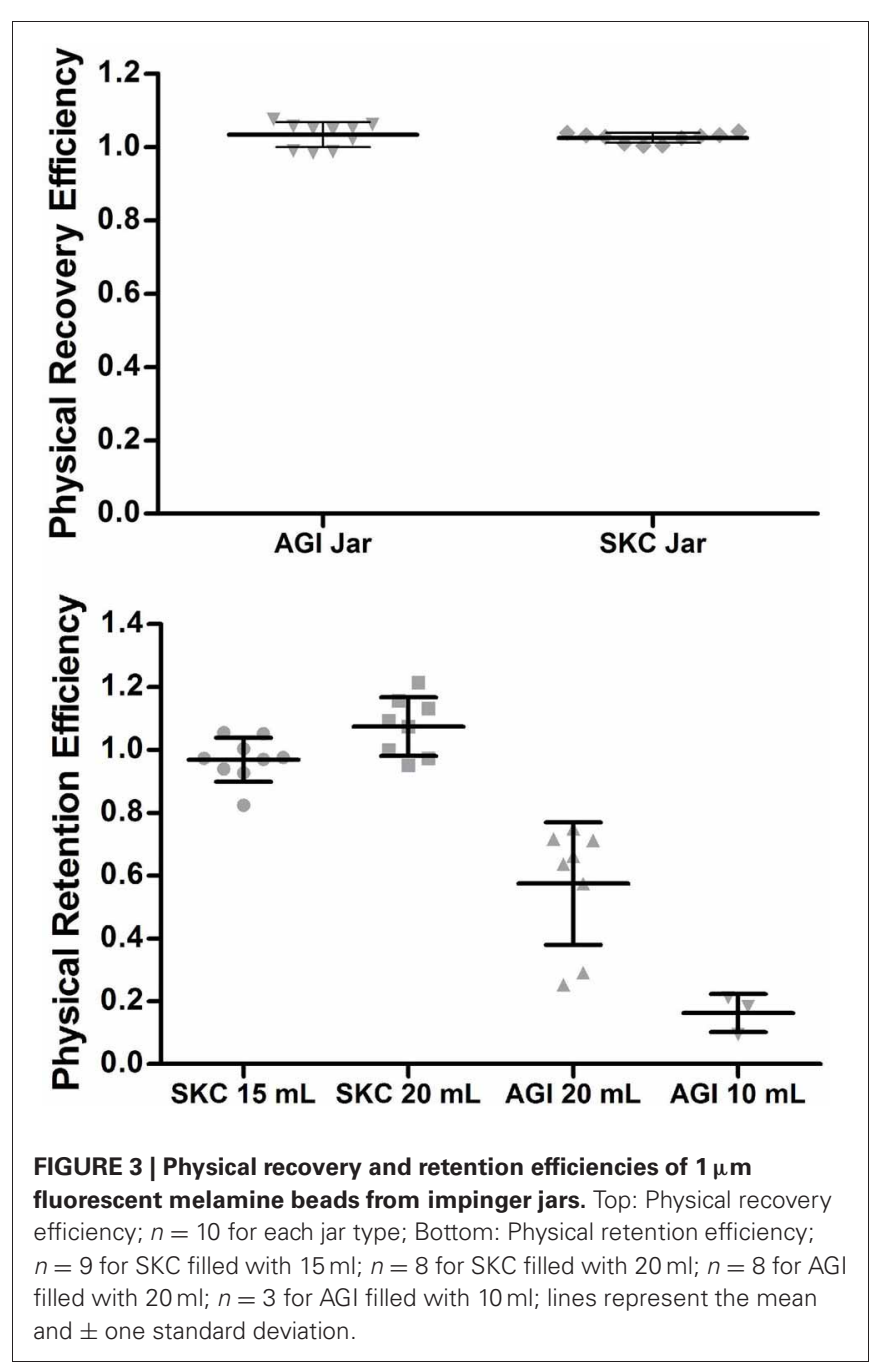

AGI jar filled with $10 \mathrm{ml}$ of media containing $B$. thailandensis was $0.79 \pm 0.08(n=6)$.

\section{DISCUSSION}

The aim of the present study was to estimate the recovery efficiency of the bacterium $B$. thailandensis from several common sampling media, specifically $25 \mathrm{~mm}$ gelatin filters, greased $25 \mathrm{~mm}$ stainless steel disks from a Mercer impactor, and jars from both an AGI and an SKC Biosampler, using a variety of different recovery processes. The results demonstrated average physical recovery efficiencies of at least 0.97 for all sampling media tested, suggesting that near complete recovery of the spiked material was possible. Several of the estimated physical retention efficiency values for the various recovery processes were greater than 1.0, with the greatest value being $1.07 \pm 0.09$ for SKC Biosampler jars filled with $20 \mathrm{ml}$ of media. However, these values are likely a result of variability associated with the fluorescence measurements, which was approximately $5 \%$ for control samples.

Several of the sampling media tested had physical recovery efficiencies that were dependent upon the recovery process utilized. For $25 \mathrm{~mm}$ gelatin filters, dissolution of the filter in

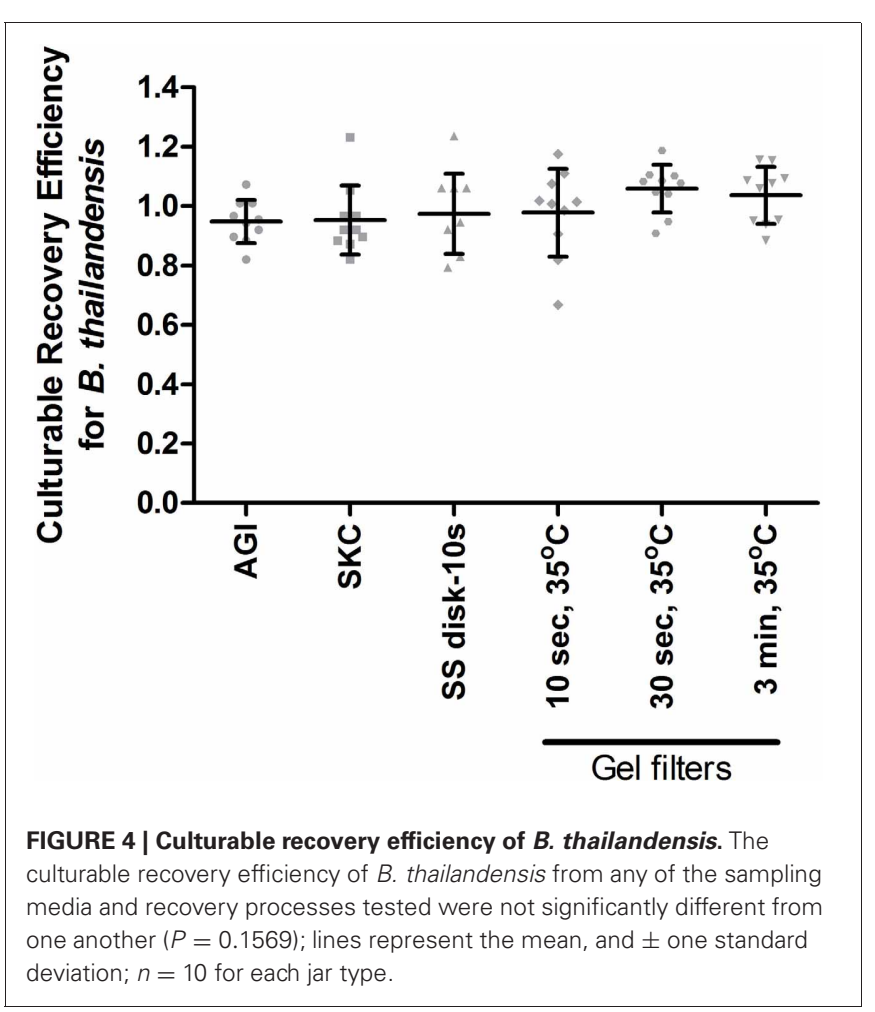

room temperature water resulted in a significantly lower recovery efficiency, and chunks of the filter were still visibly suspended even after 3 min of vortexing. Thus, the decreased physical recovery efficiency was likely due to incomplete dissolution of the filter material. For greased $22 \mathrm{~mm}$ stainless steel disks, the physical recovery efficiency decreased significantly as the duration of vortexing increased. It was noted that the walls of the conical tube became more opaque the longer the sample was vortexed, suggesting that the loss was due to binding of the fluorescent beads to the wall of the conical tube. This change in the opacity of the walls of the conical tube was unique to the greased $25 \mathrm{~mm}$ disks, and was not observed with the other sampling media tested, suggesting that some unique component of the sampling medium, potentially the grease from the surface of the disk, was enhancing the binding of the fluorescent beads to the wall of the conical tube. However, additional testing is needed to verify this hypothesis.

Estimation of the culturable recovery efficiency was not performed using processing methods that had physical recovery efficiencies that were significantly less than the other processes. Regardless, it was possible to achieve culturable recovery efficiencies near unity for all of the sampling media tested, suggesting that the recovery processes utilized were not damaging the bacteria. Some of the estimated culturable retention efficiency values for gelatin filters were greater than 1.0, with the greatest value being $1.06 \pm 0.08$ for gelatin filters vortexed for $30 \mathrm{~s}$ in $35^{\circ} \mathrm{C}$ media. These values are likely a result of variability associated with the triplicate plate counts, which was slightly less than $10 \%$. These results complement the findings of Zhao et al. (2011), in which four different bacteria spiked onto agar plates of an Andersen impactor were recovered with recovery efficiencies of $87-124 \%$. 
It should be stressed that these results are only applicable to recovery from sampling media, and that it is possible that physical or culturable losses of airborne bacteria may still occur during either transport through the sampler prior to collection or the collection process into the sampling medium. It should also be noted that there is a potential for differences in the fitness of bacteria straight from a liquid culture, like those used in the present study, and those having undergone aerosolization. Bacteria undergoing the process of aerosolization may be damaged and, therefore, less likely to survive the post-collection processing method than bacteria taken straight from a liquid culture, resulting in a reduced culturable recovery efficiency. The effect of the generation process on bacterial fitness is, therefore, a potentially important consideration in the design of a study. The effect would also likely be bacterium and generator specific, requiring additional testing for every combination utilized.

For the jars from the Model 7541 AGI and SKC Biosampler, it was possible to completely recover both the beads and B. thailandensis initially pipetted into each type of jar if they were recovered without activating the sampler. However, if the Model 7541 AGI was activated and the liquid in the jar agitated, the initial material present in the jar was not completely retained. This was observed for both $1 \mu \mathrm{m}$ fluorescent beads and for B. thailandensis. These data are similar to those reported by Kesavan et al. (2010), in which it was demonstrated that the AGI-30 did not completely retain $1 \mu \mathrm{m}$ spheres or the bacteria Bacillus atropheus and Pantoea agglomerans in the sampling medium, with losses potentially resulting from both re-aerosolization and decreases in the culturability of collected bacteria. Similarly, it has been recently reported that the retention efficiency of $B$. pseudomallei, which would be expected to behave similarly to B. thailandensis, in an Ace Glass Model 7541 All Glass Impinger is significantly less than unity (Dabisch et al., 2012), with similar mechanisms responsible for the observed losses. In the present study, it is again likely that similar mechanisms, including re-aerosolization of material from the sampling medium; binding of material to the inner walls of the jar, as is suggested by the presence of a residue on the jar; or, in the case of $B$. thailandensis, loss of viability due to the stresses associated with the sampler, are responsible for the decreased retention efficiency. However, additional testing is needed to determine the contribution of each possible mechanism to the observed loss of material. Regardless of the mechanism(s) responsible, these data suggest that additional processing steps may be necessary to completely recover collected material from Model 7541 AGIs.

The study by Kesavan et al. (2010) also demonstrated a decreased retention of both $1 \mu \mathrm{m}$ spheres and bacteria in the SKC

\section{REFERENCES}

Brockman, J. E. (2011). "Aerosol transport in sampling lines and inlets," in Aerosol Measurement: Principles, Techniques, and Applications, eds P. Kulkarni, P. A. Baron, and K. Willeke (Hoboken: Wiley and Sons), 69-105.

Cheng, A. C., and Currie, B. J. (2005). Melioidosis: epidemiology, pathophysiology, and management. Clin. Microbiol. Rev. 18, 383-416.

Currie, B. J. (2008). Advances and remaining uncertainties in the epidemiology of Burkholderia pseudomallei and melioidosis. Trans. $R$. Soc. Trop. Med. Hyg. 102, 225-227.

Dabisch, P., Yeager, J., Kline, J., Klinedinst, K., Welsch, A., and Pitt, M. L. (2012). Comparison

Biosampler over time. These results are contrary to the results reported in the present study in which $1 \mu \mathrm{m}$ spheres were completely retained in the SKC Biosampler following a $10 \mathrm{~min}$ period of sampler air flow. The difference between the results of these studies may result from the different durations of sampler air flow used - the present study utilized a $10 \mathrm{~min}$ period of sampler air flow, whereas Kesavan et al. utilized 15, 20, 45, and 60 min durations of sampler air flow. It is possible that the $10 \mathrm{~min}$ period utilized in the present study was not sufficiently long to produce a significant loss of beads from the sampling medium.

As noted previously, it is important to have knowledge of the various efficiencies associated with the sampling process, especially in studies that require calculation of the absolute concentration of airborne material, such as inhalation dosing studies. Erroneous assumptions regarding sampling efficiency values have the potential to result in miscalculation of the airborne concentration of microorganisms, and ultimately affecting any subsequent estimations of risk or dose derived from such data. The data from the present study provide novel information on the recovery and retention efficiencies for a bacterium commonly used in inhalation studies from commonly used sampling devices, demonstrating that the mechanism of operation of the sampling device as well as the post-collection processing method can significantly affect the recovery and retention of culturable microorganisms from sampling media. These data will useful to inform sampling paradigms in future inhalation studies, and may also be applicable to other similar bacteria, notably B. pseudomallei, for which inhalation has been suggested as a natural route of exposure.

\section{DISCLAIMER}

This manuscript was developed and funded under Agreement No. HSHQDC-07-C-00020 awarded by the US Department of Homeland Security for the management and operation of the National Biodefense Analysis and Countermeasures Center (NBACC), a Federally Funded Research and Development Center. The views and conclusions contained in this document are those of the authors and should not be interpreted as necessarily representing the official policies, either expressed or implied, of the US Department of Homeland Security. The Department of Homeland Security does not endorse any products or commercial services mentioned in this publication. Opinions, interpretations, conclusions, and recommendations are those of the author and are not necessarily endorsed by the National Biodefense Analysis and Countermeasures Center (NBACC), Department of Homeland Security (DHS), or Battelle National Biodefense Institute (BNBI).

of the efficiency of sampling devices for aerosolized Burkholderia pseudomallei. Inhal. Toxicol. 24, 247-254.

Haraga, A., West, T. E., Brittnacher, M. J., Skerrett, S. J., and Miller, S. I. (2008). Burkholderia thailandensis as a model system for the study of the virulence-associated type III secretion system of Burkholderia pseudomallei. Infect. Immunol. 76, 5402-5411.

Inglis, T. J., and Sagriapanti, J. L. (2006). Environmental factors that affect the survival and persistence of Burkholderia pseudomallei. Appl. Environ. Microbiol. 72, 6865-6875.

Kesavan, J., Schepers, D., and McFarland, A. R. (2010). Sampling and retention efficiencies of 
batch-type liquid-based bioaerosol samplers. Aerosol Sci. Tech. 44, 817-829.

Morici, L. A., Heang, J., Tate, T., Didier, P. J., and Roy, C. J. (2010). Differential susceptibility of inbred mouse strains to Burkholderia thailandensis aerosol infection. Microb. Pathog. 48, 9-17.

Reponen, T., Willeke, K., and Grinshpun, S. (2011). "Biological particle sampling," in Aerosol Measurement: Principles, Techniques, and Applications, eds $\mathrm{P}$. Kulkarni, P. A. Baron, and K. Willeke (Hoboken: Wiley and Sons), 549-570.

Rozak, D. A., Gelhaus, H. C., Smith, M., Zadeh, M., Huzella, L., Waag, D., and Adamovicz, J. J. (2010). CpG oligodeoxyribonucleotides protect mice from Burkholderia pseudomallei but not Francisella tularensis Schu S4 aerosols. J. Immune Based Ther. Vaccines 8, 1-5.

Sivalingam, S. P., Sim S. H., Jasper, L. C., Wang, D., Liu, Y., and Ooi, E. E. (2008). Pre- and post-exposure prophylaxis of experimental Burkholderia pseudomallei infection with doxycycline, amoxicillin/clavulanic acid and co-trimoxazole. J. Antimicrob. Chemother. 61, 674-678.

Tan, G. G., Liu, Y., Sivalingam, S. P., Sim, S., Wang, D., Paucod, J., Gauthier, Y., and Ooi, E. (2008) Burkholderia pseudomallei aerosol infection results in differential inflammatory responses in $\mathrm{BALB} / \mathrm{c}$ and $\mathrm{C} 57 \mathrm{Bl} / 6$ mice. J. Med. Microbiol. 57, 508-515.

West, T. E., Frevert, C. W., Liggitt, H. D., and Skerrett, S. J. (2008).
Inhalation of Burkholderia thailandensis results in lethal necrotizing pneumonia in mice: a surrogate model for pneumonic melioidosis. Trans. R. Soc. Trop. Med. Hyg. 102, S119-S126.

Zhao, Y., Aarnink, A. J. A., Doornenbal, P., Huynh, T. T. T., Koerkamp, P. W. G., Groot, D., Mart, C. M., and Landman, W. J. M. (2011). Investigation of the efficiencies of bioaerosol samplers for collecting aerosolized bacteria using a fluorescent tracer. I: effects of nonsampling processes on bacterial culturability. Aerosol Sci. Technol. $45,423-431$.

Conflict of Interest Statement: The authors declare that the research was conducted in the absence of any commercial or financial relationships that could be construed as a potential conflict of interest.

Received: 16 February 2012; paper pending published: 30 March 2012; accepted: 21 May 2012; published online: 11 June 2012.

Citation: Dabisch P, Bower K, Dorsey B and Wronka L (2012) Recovery efficiencies for Burkholderia thailandensis from various aerosol sampling media. Front. Cell. Inf. Microbio. 2:78. doi: 10.3389/ fimb. 2012.00078

Copyright (c) 2012 Dabisch, Bower, Dorsey and Wronka. This is an openaccess article distributed under the terms of the Creative Commons Attribution Non Commercial License, which permits non-commercial use, distribution, and reproduction in other forums, provided the original authors and source are credited. 